\title{
Mechatronics Design of an Autonomous Pipe-Inspection Robot
}

\author{
Mohamed Abdellatif ${ }^{1}$, Hazem Mohamed ${ }^{2}$, Mohamed Hesham ${ }^{2}$, Ahmed Abdelmoneim $^{2}$, Amro Kamal $^{2}$ \\ and Ahmed Khaled ${ }^{2}$ \\ ${ }^{1}$ Future University in Egypt, Mechanical Eng. Department, Cairo, Egypt \\ ${ }^{2}$ Arab Academy for Science, Technology and Maritime Transport, Cairo, Egypt
}

\begin{abstract}
Pipelines require periodical inspection to detect corrosion, deformation and congestion with obstacles in the network. Autonomous mobile robots are good solutions for this task. Visual information from the pipe interior associated with a location stamp is needed for inspection. In this paper, the previous designs of autonomous robots are reviewed and a new robot is developed to ensure simple design and smooth motion. Images are processed online to detect irregularity in pipe and then start capturing high resolution pictures to conserve the limited memory size. The new robot moves in pipes and provides video stream of pipe interior with location stamp. The visual information can later be processed offline to extract more information of pipeline condition to make maintenance decisions.
\end{abstract}

\section{Introduction}

Pipelines are important for economic transport of gases and liquids such as oil, fuels, water and sewer. However, pipelines always suffer from aging and damages, which can cause great waste of resource, environmental pollution, and other accidents. For example, the leak of petroleum pipeline causes financial losses, pollution and ecocatastrophe.

Regular inspections and maintenance of pipelines are essential to keep them functional. Unfortunately, the difficulty and the cost for human inspection can be extremely high, especially with the appearance of increasingly complicated pipelines nowadays [1]. Autonomous inspection and monitoring of pipes is important during several phases including the commissioning stage to ensure that pipes are free from faults of left-over obstacles. Then, monitoring is also needed in the running and routine maintenance phases.

Autonomous robots can be used to inspect the interior of pipes. Sewage pipelines are the most commonly inspected as it is very hard to inspect by humans [2]. Information such as pictures of cracks or any notches in the pipes [3] need to be collected. It may also include videos showing the internal shape of pipes, bends and manholes. Location of the cracks, inside the pipelines, is extremely important for repair. A fully autonomous robot is needed to relax human intervention that is boring and hard to maintain in high quality and attention for longer periods of time. Autonomous robots are needed for inspection because pipeline systems are often hidden underground and are long, thin, and complex. Furthermore, the environment inside pipelines can be dirty and hazardous for humans. Several systems were built in the world to inspect pipe interiors $[4,5]$. The classification can be based on several criteria such as autonomous versus manual and tethered versus untethered. The current technology can mainly be classified into three categories according to the degree of robot autonomy: namely; remotely operated, semiautonomous and fully autonomous. All three categories are tethered in general while the third category can be tethered or not $[6,7]$.

Although several robots had been introduced in the literature, the mechatronics design is complex and is not easy to operate besides the high cost of the robot $[8,9]$. In general the complexity resulted from the requirements to satisfy the projects needs in building such robots, including the ability to climb steep obstacles or to navigate through pipe networks of curved routes and connections. An autonomous robot that can routinely inspect the pipe interior and report deformation will, therefore, be highly desirable for routine maintenance [10-12].

In this paper, we describe the design and development of an autonomous robot capable of inspecting pipes interior of $30 \mathrm{~cm}$ diameter which constitute around $60 \%$ of pipes used in sewage systems in several countries, including Egypt and Japan [2]. The design objective is to build a simple system and can be made from off the shelf components.

A user interface program for offline visual information processing is also developed in this work to help the users in interpreting the pipe data and customizing their image processing on images and video streams.

This paper is arranged as follows: in the following section, the literature and background will be briefly reviewed showing a number of famous inspection robots and comparing them. In section 3 , the design of the new 
system is considered in view of the system requirements and the motivation for the design parameters and selections are discussed. In section 4, experiment with the real robot is presented and the GUI design for easy operation by users is presented. Finally, conclusions are given in section 5 summarizing the obtained results.
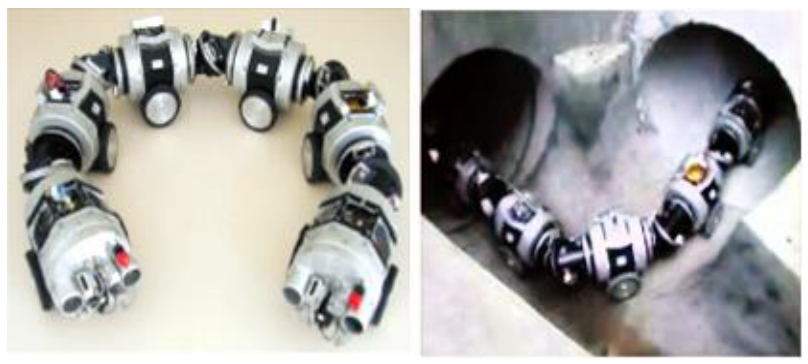

Figure 1. MAKRO a) photograph, b) turning
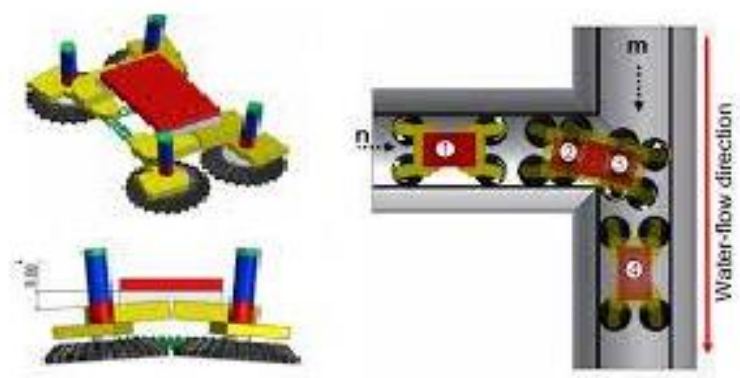

Figure 2. a) Kantaro structure and b) turning in bends
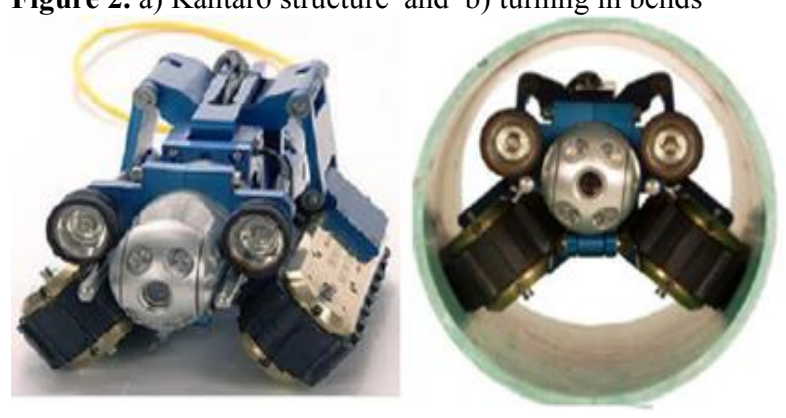

Figure 3. Versatrax system.

\section{Background}

MAKRO, [4] is an articulated inspection robot of wormlike shape and is designed for autonomous navigation in sewer pipes of 30 to $60 \mathrm{~cm}$ width. Its design, as shown in Fig. 1, consists of six segments connected by five motordriven active joints, allows for simultaneously climbing a step and turning, at a junction consisting of a $600 \mathrm{~mm}$ pipe diameter and a branching $300 \mathrm{~mm}$ pipe diameter with equal top levels. MAKRO carries all the needed resources on-board and can run for two hours. It has to find and identify manholes and junctions; must be able to turn within manholes up to 90 degrees. The robot can 1) go to a specified point in the sewer system, 2) scan/inspect area and 3) get water sample.

Each joint has three infrared angle decoders and an odometer for the wheels. In each head four infrared sensors are used for distance measurement to the sides, up and down. Ultrasonic sensor detects obstacles in the frontal direction. This robot in our opinion is expensive and complexity is more than needed for the task complexity.

KANTARO, [1] is an autonomous, un-tethered robot, which fits to pipe diameter range of 200-300 $\mathrm{mm}$. Kantaro [12] consists of a bottom box, including a passive-active mechanism and a battery pack, can be presented as a robot platform. The upper box contains the electronic board, the sensors and light connected via the main connector to the bottom box as shown in Fig.2. KANTARO has a passive active mechanism that can move through straight and curved

pipes without sensors or controllers. The design is based on passive adaptation of robot wheels to the bends in the pipe through combined wheels orientation and passive damping of springs.

Kantaro mechanism has four horizontal wheels connected to the four arms and each arm is jointed to the base plate, independently, and they are connected to each other by using four springs.

The laser scanner and fisheye camera are used for inspection and fault-navigation. Two IR sensors are installed in front of robot in two side of the camera to avoid driving the robot in a step down more than $10 \mathrm{~cm}$ and protect against obstacles. Rotating laser scanner has been developed and installed in the robot rear to scan radially the inner side of a pipe wall. Kantaro can detect the candidate faulty images on-board, using a fast and simple edge detection program. When robot detects a feature-image, an autonomous control program will start to reduce the robot speed for capturing more images, up to 5 frames, from feature image area. At this time, the laser scanner, that is normally switched off will start to recognize the feature faults as a landmarks. The captured images are transferred to an offline station to find faulty images and estimate their location.

The robot system is complicated because of the need to pass through different successive bends and changing pipe diameters. The case is not with such complexity in several other countries because of high housing density in Japan. It is simpler to develop robots that can work for specific and popular pipe sizes with straight configuration.

The Versatrex robot, shown in Fig.3, has a compact design and uses tracks to move straight in pipes.

\section{Mechatronics System Design}

The mechanism must enter into the internal pipe diameter $300 \mathrm{~mm}$ which is the constraint for robot outer dimensions. According to literature survey, $60 \%$ of sewage pipes have diameter of $300 \mathrm{~mm}$.

Sewage pipelines are predicted to be full of sewerage content especially at the bottom. Consequently, the robot base should not touch the pipe bottom. We selected ready-made robot with tracked wheel and split the base of the robot. The two tracks equipped with motors are arranged to form part of two points passing through the arc of pipe with $300 \mathrm{~mm}$ diameter. The two tracks were linked by an aluminium base as shown in Fig 4. A casing had been made from acrylic to cover this base and to protect the sensor and computing devices from water or 
other hazardous materials, as shown in Fig.5. The final mechanical design is shown in Fig. 6.

A front ultrasonic sensor is used to detect obstacles. A video camera module is also fitted on robot front to capture the video stream and report it to control module which archive the video stream for retrieval and process on board to detect abnormality. The camera is special to Rasberry PI and have five megapixels sensor array supporting resolution of 1080, 720 and VGA video modes. The camera is attached via a $15 \mathrm{~cm}$ ribbon cable to the CSI port on the raspberry module. This camera has high resolution and in same time small and appropriate to our robot. Raspberry pi camera module was found appropriate to our robot, since it can be used to take highdefinition video, as well as stills photograph

In case abnormal images are detected then the camera is directed to capture high resolution images for further offline analysis. Encoders are fitted to the DC motors of the tracks to show the travelled distance. The quadrature encoder has resolution of 1000 state change per wheel cycle. Two dc motors with motor torque of $10 \mathrm{~kg} . \mathrm{cm}$ are used. A lithium battery is also selected for two hours operation.

Raspberry PI was selected since compared to other options; it excels in handling video and multimedia data in general. Raspberry Pi was chosen for several reasons:

-Uses ARM processor with strong computing capability.

-Easy to program in scripting python.

- Low power consumption to increase mission time.

- Has optional memory module to enable storage of video and still images.

-Processing saved data.

The board have SD Card slot for data storage up to 32 G-Byte which is sufficient for expected two hour missions.

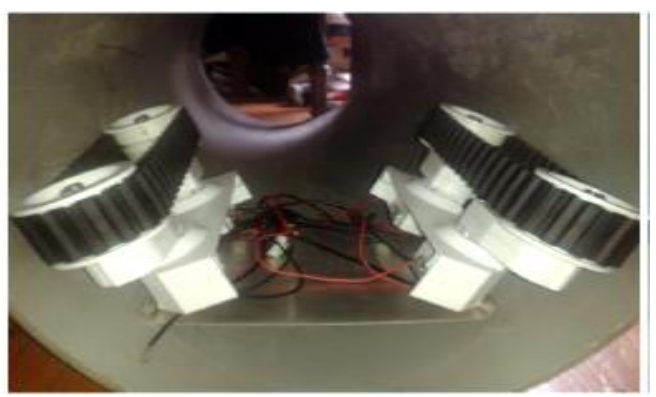

Figure 4. Educational Rover bottom view, it was split into two parts with horizontal cutting section to build the new inspection robot, lower picture shows new arrangement.

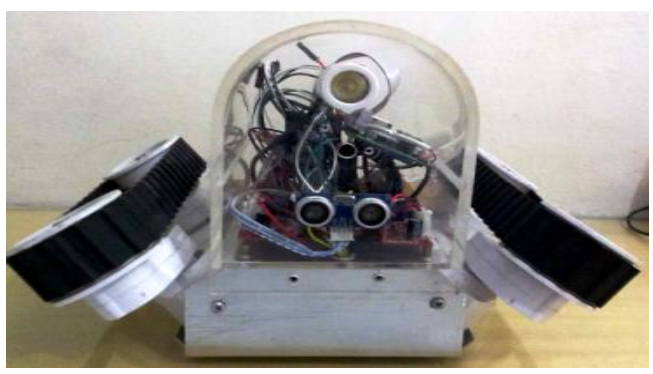

Figure 5. The final robot assembly.
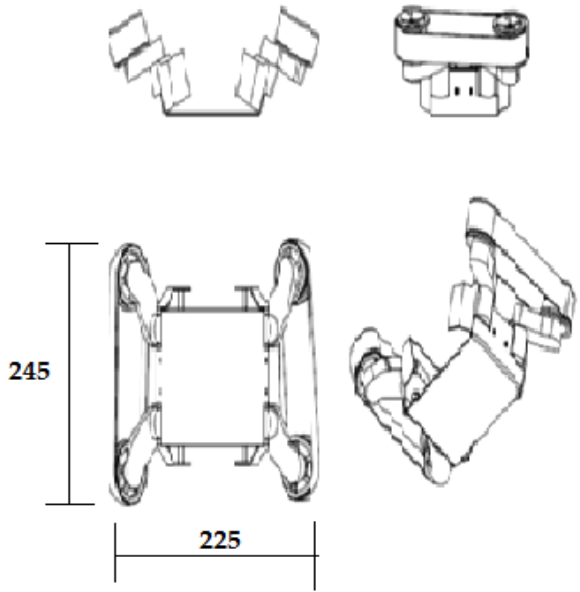

Figure 6. Mechanical Design of the proposed pipe inspection robot.

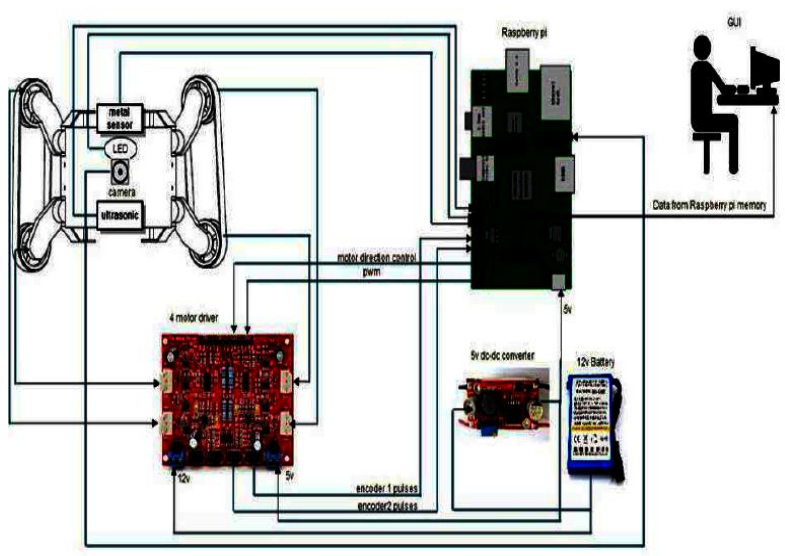

Figure 7. Schematic representation of the pipe inspection robotic system.

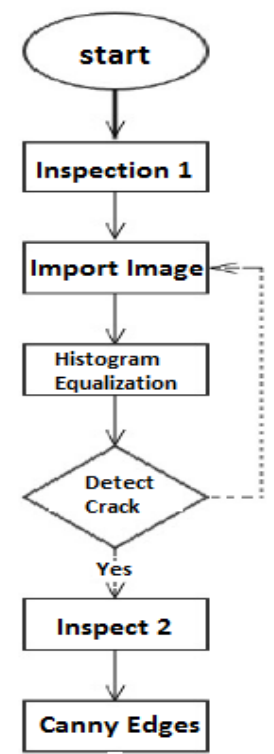

Figure 8. The flow chart of image processing operations.

\section{Experiments}

The robot is shown while tested to fit in pipe as in Fig.4. The complete system with assembled sensors and computing is shown in Fig. 7. 
The mechanical chassis of robot is assembled and motors tested to move and fit in pipe of $30 \mathrm{~cm}$ internal diameter. The Raspberry acts as the computing brain of robot and responsible for motion commands to track motors through an interface, then it reads signal from the motor encoders. The Raspberry also controls the operation of the camera to capture whether video streams or high resolution still images. The videos or images are stored in memory storage during autonomous operation of robot in pipes. After the robot mission in pipe is over, the robot is retrieved and the memory is used by an offline user with analysis program that can read the images, videos and location stamps of the visual data.

The robot was tested to move inside a test pipe section without defects. The external interface program allows the user to read both still images and videos. It contain image enhancement option by using the Histogram Equalization, HE, techniques. This had the effect of improving the image clarity for user especially for dim lighting. Other color enhancement techniques can be used as well [13] and planned in future.

Objective of robot is to provide the interface for retrieval of visual information collected during the mission of pipe inspection. The location of the robot is written in the title of saved images and videos so that for a specific mission, the robot location inside the pipe relative to pipe entrance location is known. The location information relies now on encoder information only. We intend to use a version of visual SLAM algorithm so that we can have another source for localization inside the pipe. Currently the encoder reading will have accumulated errors which increase with the length of robot mission.

The flow chart of image processing is shown in Fig. 8. This program is executed online during robot mission. Program interface is shown in Fig. 9. It is used to enhance image ( histogram equalization) and detect edges ( Canny algorithm) ( inspect 1 and 2).

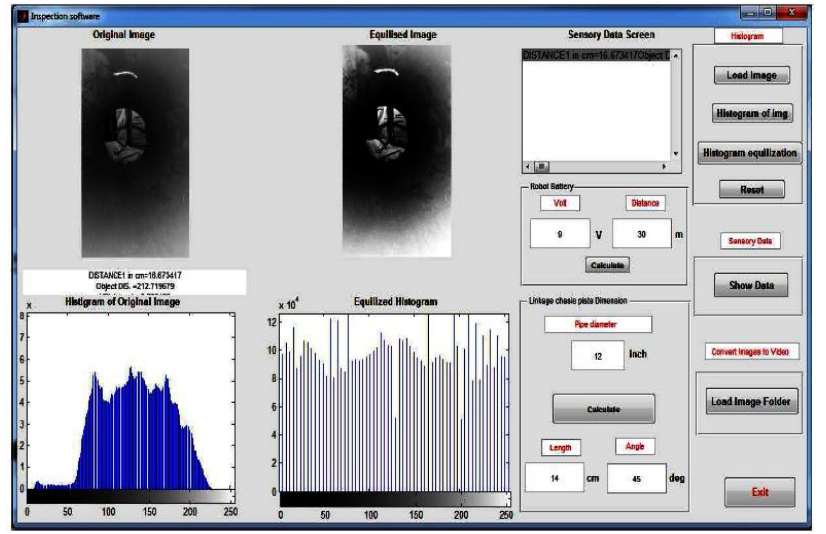

Figure 9. The user interface showing options to display original and enhanced images and showing histogram or other useful statistics (inspect 1 software).

Matlab was used to build a custom GUI. The robot captures live video during its motion in pipe and detects any defect automatically using edge detection algorithm. A flag is raised when detecting abnormality to save still high resolution image in defected areas. When the robot mission is over, the memory card is restored and attached to an offline computer. Paragon software is used to read files from memory and data is imported online by filezilla program.

The offline processing was made by custom developed software with all requirements of user in mind. The GUI shows the images and defect location and offer a variety of useful image processing operation to be customized by the user.

In Fig. 10 is shown the original captured image with intentional crack in the pipe shown in a and in $b$ is shown its enhanced version where the crack image have better contrast. The edge contour can be used to check any irregular pipe deformation when compared with a normal circle or ellipse with smooth trajectory. Further processing like this is left for other dedicated image processing software which already exists.

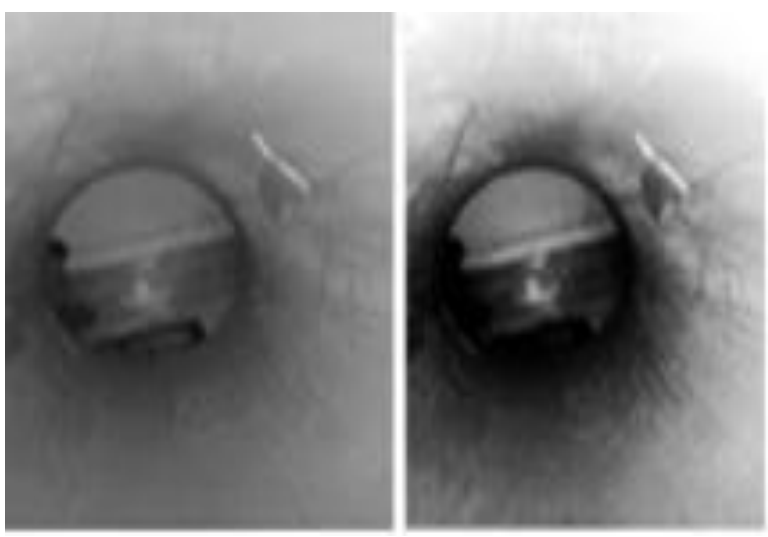

Figure 10. The pipe images showing a) a crack, b) Enhanced image.

\section{Conclusions}

Since inspection of pipelines is hard for a human inspector, an autonomous robot is needed for this task. We designed a new autonomous robot in consideration of pros and cons of the previous arts. Our mechatronics design is justified through comparison among best available modules and components. The control system is devised to ensure smooth operation of the robot. The new robot captures images and videos of the suspected cracks. It can also detect the location and orientation of the pipe defects between two certain manholes.

\section{References}

1. A.F. Nassiraei, Y. Kawamura, A. Ahrary, Y. Mikuriya, and K. Ishii, Concept and design of a fully autonomous sewer pipe inspection mobile robot "KANTARO", Proceedings of the IEEE International Conference on Robotics and Automation (ICRA'07), pp.136-143, 2007.

2. A. Ahrary, Y. Kawamura, and M. Ishikawa, A study of an autonomous mobile robot for a sewer inspection system, Journal of Artificial Life and Robotics, Vol.11, No.1, pp.23-27, January 2007. 
3. H. Alireza, G. Hadi, and M. Hossein, Developing a sewer inspection robot through a mechatronics approach, International Conference on Mechanical, Automotive and Materials Engineering (ICMAME'2012), 2012 Dubai, pp.187-191.

4. E. Rome, J. Hertzberg, F. Kirchner, U. Licht, H. Streich, and TH. Christaller, Towards autonomous sewer robots: The MAKRO project, Journal of Urban Water, Vol.1, No.1, pp. 57-70, March 1999.

5. H. R. Choi and S. M. Ryew, Robotics system with active steering capability for internal inspection of urban gas pipelines, Mechatronics, vol.26, no.1, pp.105-112, 2002.

6. L. Hun-ok and O. Taku, Development of pipe inspection robot, ICROS-SICE International Joint Conference 2009.

7. K. Yun-Jong, Y. Kyung-Hyun and P. Young-Woo, Development of In-pipe robot for various sizes, ASME International Conference on Advanced Intelligent Mechatronics Suntec Convention and Exhibition Center Singapore, 2009.

8. K. U. Scholl, V. Kepplin, K. Berns and R. Dillmann, Controlling a multi-joint robot for autonomous sewer inspection, Proceedings of IEEE/RAS International Conference on Robotics and Automation, ICRA 2000, pp. 1701-1706.

9. R. Kirkham, P. D. Kearney and K. J. Rogers, PIRAT - A system for quantitative sewer assessment, The International Journal of Robotics Research, Vol.19, No.11, pp. 1033- 1053, 2000.

10. M. Moghaddam, A. Hadi and T. Abbasian, Design and Manufacturing SEWBOT: a Sewer Inspection Robot, 1st International Conference on Technical Inspection and NDT, October 2007, Tehran, Iran.

11. O. Tatar, D. Mandru and I. Ardelean, Development of mobile minirobots For in pipe inspection tasks, Mechanik, No..6(68) pp. 60-64, 2007.

12. S.G. Roh, S. M. Ryew, J. H. Yang and H. R. Choi, Actively steerable in pipe inspection robots for underground urban gas pipelines, IEEE International Conference on Robotics \& Automation, Korea, 2001.

13. M. Abdellatif, Effect of color preprocessing on color based object detection, Proceeding of SICE annual conference, pp:1124-1129, 2008. 